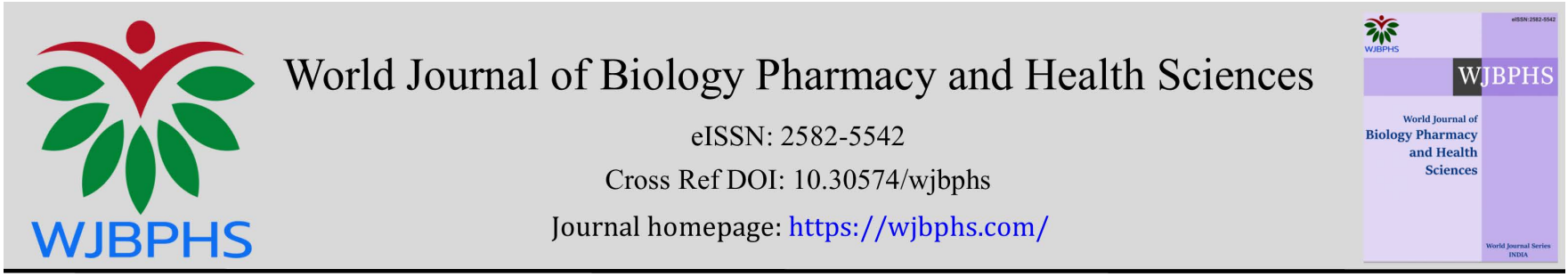

(RESEARCH ARTiCLE)

\title{
Pollutants and climatic conditions related to the smoking rate
}

\author{
Shigeru Suna * \\ Private Health Research Laboratory, 14-22 Shinkita-machi, Takamatsu-shi, Kagawa 760-0001, Japan.
}

World Journal of Biology Pharmacy and Health Sciences, 2021, 08(02), 034-041

Publication history: Received on 07 October 2021; revised on 25 November 2021; accepted on 27 November 2021

Article DOI: https://doi.org/10.30574/wjbphs.2021.8.2.0119

\begin{abstract}
Smoking is considered a coping behavior for stress. On the other hand, pollutants and climatic conditions are environmental stressors that can have a negative impact on health. Therefore, there may be a link between regional smoking rate and air pollution and climatic conditions.

To clarify the relationship between the regional smoking rate in Japan and the environmental conditions such as photochemical oxidants concentration, ambient temperature and relative humidity, multiple regression analysis was performed.

Correlation analysis showed that the ambient temperature and relative humidity and photochemical oxidants are negatively correlated with smoking rate. Stepwise multiple regression analysis with smoking rate as the objective variable, the ambient temperature and relative humidity and photochemical oxidants as explanatory variables, revealed that the ambient temperature and relative humidity and photochemical oxidants are significant independent variables.

The above results suggest that ambient temperature, relative humidity and photochemical oxidants in the region may be related to smoking rate in the region.
\end{abstract}

Keywords: Smoking rate; Photochemical oxidants; Ambient temperature; Relative humidity

\section{Introduction}

Smoking is considered a coping behavior for stress [1-4]. On the other hand, pollutants and climatic conditions are environmental stressors that can have a negative impact on health [5-7]. Therefore, there may be a link between regional smoking rate and air pollution and climatic conditions.

Since the Japanese archipelago is long from north to south, it belongs to various climate categories, from the subarctic zone in the north to the subtropical zone in the south. In addition, because the Japanese archipelago has mountain ranges with high mountains, there are many cloudy, snowy or rainy days on the Sea of Japan side in winter, while there are many sunny days on the Pacific side [8]. Therefore, there are significant differences in weather depending on the region. Also, climatic differences may affect the formation of photochemical oxidants [9].

This study shows the relationship between smoking rate in Japanese prefectures and photochemical oxidants concentrations and climatic conditions.

\footnotetext{
${ }^{*}$ Corresponding author: Shigeru Suna

Private Health Research Laboratory, 14-22 Shinkita-machi, Takamatsu-shi, Kagawa 760-0001, Japan.
} 


\section{Methods}

\subsection{Smoking rate}

The smoking rate in prefectures is based on the comprehensive living conditions surveys by the Ministry of Health, Labour and Welfare. The survey is conducted every 3 years. The values were downloaded from the Cancer Information Service of the National Cancer Center. (https://ganjoho.jp/reg_stat/statistics/dl/index.html\#smoking). The smoking rate is the ratio of the total number of people (men and women over 20 years old) who answered "smoking daily" or "sometimes smoked" as a numerator and the "total number of respondents" as a denominator.

\subsection{Air pollutants}

Annual average photochemical oxidants concentrations in prefectures were obtained from the National Institute for Environmental Studies.

(https://tenbou.nies.go.jp/gis/monitor/?map_mode=jpn_env_atmosphere)

\subsection{Climatic conditions}

The annual average values of ambient temperature and relative humidity in prefectures were from Social Indicators by Prefecture. The values were downloaded from e-Stat (https://www.e-stat.go.jp/dbview?sid=0000010102). e-Stat is a portal site for Japanese Government Statistics.

\subsection{Statistical analysis}

Multiple linear regression analysis was performed to determine the relationship between smoking rate and environmental conditions. $\mathrm{p}<0.05$ was considered as statistically significant.

\section{Results}

\subsection{Smoking rate}

Table 1 shows the smoking rates of prefectures according to the 2010,2013, and 2016 comprehensive living conditions surveys.

Table 1 Smoking rate of prefectures according to the 2010,2013, and 2016 comprehensive living conditions surveys

\begin{tabular}{|l|c|c|c|l|c|c|c|}
\hline \multirow{2}{*}{ Prefecture } & \multicolumn{3}{|c|}{ Smoking (\%) } & \multirow{2}{*}{ Prefecture } & \multicolumn{3}{|c|}{ Smoking (\%) } \\
\cline { 2 - 5 } \cline { 7 - 8 } & $\mathbf{2 0 1 0}$ & $\mathbf{2 0 1 3}$ & $\mathbf{2 0 1 6}$ & & $\mathbf{2 0 1 0}$ & $\mathbf{2 0 1 3}$ & $\mathbf{2 0 1 6}$ \\
\hline Hokkaido & 24.8 & 27.6 & 24.7 & Shiga & 19.1 & 19.8 & 18.7 \\
\hline Aomori & 24.7 & 25.9 & 23.8 & Kyoto & 19.1 & 18.5 & 17.5 \\
\hline Iwate & 22.4 & 23.4 & 22.6 & Osaka & 22.3 & 22.3 & 19.9 \\
\hline Miyagi & 22.9 & 24.1 & 21.0 & Hyogo & 19.0 & 19.2 & 18.9 \\
\hline Akita & 22.5 & 23.5 & 20.3 & Nara & 18.2 & 17.0 & 17.1 \\
\hline Yamagata & 20.6 & 20.9 & 19.3 & Wakayama & 19.3 & 19.8 & 18.9 \\
\hline Fukushima & 23.0 & 25.1 & 22.4 & Tottori & 19.1 & 19.7 & 18.2 \\
\hline Ibaraki & 21.4 & 23.3 & 21.6 & Shimane & 17.3 & 19.7 & 18.0 \\
\hline Tochigi & 22.8 & 22.7 & 21.8 & Okayama & 19.6 & 19.7 & 18.8 \\
\hline Gunma & 22.8 & 23.3 & 22.0 & Hiroshima & 19.5 & 20.5 & 18.1 \\
\hline Saitama & 22.7 & 23.1 & 20.8 & Yamaguchi & 18.5 & 19.8 & 19.1 \\
\hline Chiba & 22.8 & 21.8 & 21.1 & Tokushima & 18.4 & 18.0 & 17.4 \\
\hline
\end{tabular}




\begin{tabular}{|l|c|c|c|l|c|c|c|}
\hline Tokyo & 20.3 & 20.9 & 18.3 & Kagawa & 20.2 & 19.4 & 17.4 \\
\hline Kanagawa & 22.1 & 19.8 & 20.0 & Ehime & 18.9 & 18.2 & 18.0 \\
\hline Niigata & 21.0 & 21.7 & 20.0 & Kochi & 19.9 & 21.9 & 19.3 \\
\hline Toyama & 20.4 & 19.7 & 19.5 & Fukuoka & 22.7 & 23.6 & 20.4 \\
\hline Ishikawa & 19.8 & 21.3 & 19.7 & Saga & 21.3 & 23.1 & 21.7 \\
\hline Fukui & 18.7 & 20.5 & 19.8 & Nagasaki & 20.6 & 22.3 & 18.9 \\
\hline Yamanashi & 21.7 & 23.3 & 20.5 & Kumamoto & 19.7 & 20.9 & - \\
\hline Nagano & 19.7 & 20.0 & 19.5 & Oita & 19.7 & 21.7 & 19.1 \\
\hline Gifu & 19.5 & 20.5 & 17.7 & Miyazaki & 21.1 & 21.3 & 20.0 \\
\hline Shizuoka & 20.9 & 21.7 & 19.9 & Kagoshima & 18.4 & 19.7 & 17.4 \\
\hline Aichi & 21.7 & 21.2 & 18.8 & Okinawa & 20.4 & 20.6 & 18.2 \\
\hline Mie & 20.3 & 19.4 & 17.7 & mean (SD) & \multicolumn{5}{|c}{$20.6(2.0)$} \\
\hline
\end{tabular}

\subsection{Air pollutants and climatic conditions}

Table 2, 3 and 4 show the ambient temperature, relative humidity and photochemical oxidants of the prefectures in 2010, 2013 and 2016.

Table 2 Annual average values of ambient temperature by prefecture

\begin{tabular}{|l|c|c|c|l|c|c|c|}
\hline \multirow{2}{*}{ Prefecture } & \multicolumn{3}{|c|}{ Ambient temperature $\left({ }^{\circ} \mathbf{C}\right)$} & Prefecture & \multicolumn{3}{|c|}{ Ambient temperature $\left({ }^{\circ} \mathbf{C}\right)$} \\
\cline { 2 - 4 } & $\mathbf{2 0 1 0}$ & $\mathbf{2 0 1 3}$ & $\mathbf{2 0 1 6}$ & & $\mathbf{2 0 1 0}$ & $\mathbf{2 0 1 3}$ & $\mathbf{2 0 1 6}$ \\
\hline Hokkaido & 9.8 & 9.2 & 9.3 & Shiga & 15.3 & 15.1 & 15.8 \\
\hline Aomori & 11.1 & 10.5 & 11.0 & Kyoto & 16.4 & 16.2 & 17.1 \\
\hline Iwate & 11.0 & 10.6 & 11.2 & Osaka & 17.3 & 17.1 & 17.7 \\
\hline Miyagi & 13.2 & 12.7 & 13.5 & Hyogo & 17.2 & 17.0 & 17.8 \\
\hline Akita & 12.3 & 11.9 & 12.5 & Nara & 15.4 & 15.3 & 16.0 \\
\hline Yamagata & 12.5 & 11.9 & 12.7 & Wakayama & 17.1 & 17.0 & 17.7 \\
\hline Fukushima & 13.8 & 13.4 & 14.2 & Tottori & 15.6 & 15.5 & 16.0 \\
\hline Ibaraki & 14.5 & 14.3 & 14.8 & Shimane & 15.5 & 15.4 & 15.9 \\
\hline Tochigi & 14.8 & 14.4 & 14.8 & Okayama & 16.7 & 16.4 & 16.6 \\
\hline Gunma & 15.5 & 15.2 & 15.6 & Hiroshima & 16.6 & 16.6 & 17.2 \\
\hline Saitama & 15.8 & 15.6 & 15.9 & Yamaguchi & 16.0 & 15.8 & 16.5 \\
\hline Chiba & 16.6 & 16.6 & 16.8 & Tokushima & 17.0 & 16.8 & 17.6 \\
\hline Tokyo & 16.9 & 17.1 & 16.4 & Kagawa & 17.0 & 16.8 & 17.5 \\
\hline Kanagawa & 16.5 & 16.6 & 16.9 & Ehime & 16.9 & 16.8 & 17.6 \\
\hline Niigata & 14.4 & 13.8 & 14.5 & Kochi & 17.6 & 17.3 & 18.1 \\
\hline Toyama & 14.9 & 14.5 & 15.2 & Fukuoka & 17.5 & 17.7 & 18.1 \\
\hline Ishikawa & 15.1 & 15.0 & 15.7 & Saga & 17.0 & 17.1 & 17.9 \\
\hline Fukui & 15.0 & 14.9 & 15.6 & Nagasaki & 17.5 & 17.5 & 18.1 \\
\hline
\end{tabular}


World Journal of Biology Pharmacy and Health Sciences, 2021, 08(02), 034-041

\begin{tabular}{|l|l|l|l|l|l|l|l|}
\hline Yamanashi & 15.2 & 15.4 & 15.7 & Kumamoto & 17.4 & 17.2 & 18.0 \\
\hline Nagano & 12.6 & 12.3 & 13.1 & Oita & 17.0 & 16.9 & 17.6 \\
\hline Gifu & 16.4 & 16.3 & 16.9 & Miyazaki & 17.7 & 17.9 & 18.6 \\
\hline Shizuoka & 17.2 & 17.2 & 17.6 & Kagoshima & 18.9 & 18.9 & 19.6 \\
\hline Aichi & 16.6 & 16.4 & 17.0 & Okinawa & 23.1 & 23.3 & 24.1 \\
\hline Mie & 16.6 & 16.5 & 16.9 & mean (SD) & \multicolumn{3}{|c|}{$15.9(2.4)$} \\
\hline
\end{tabular}

Table 3 Annual average values of relative humidity by prefecture

\begin{tabular}{|l|c|c|c|l|c|c|c|}
\hline \multirow{2}{*}{ Prefecture } & \multicolumn{2}{|c|}{ Relative humidity (\%) } & Prefecture & \multicolumn{3}{|c|}{ Relative humidity (\%) } \\
\cline { 2 - 4 } & $\mathbf{2 0 1 0}$ & $\mathbf{2 0 1 3}$ & $\mathbf{2 0 1 6}$ & & $\mathbf{2 0 1 0}$ & $\mathbf{2 0 1 3}$ & $\mathbf{2 0 1 6}$ \\
\hline Hokkaido & 69 & 71 & 66 & Shiga & 74 & 72 & 73 \\
\hline Aomori & 75 & - & 76 & Kyoto & 64 & 64 & 66 \\
\hline Iwate & 74 & 77 & 74 & Osaka & 62 & 61 & 65 \\
\hline Miyagi & 72 & 71 & 68 & Hyogo & 68 & 63 & 65 \\
\hline Akita & 74 & 74 & 74 & Nara & 73 & 72 & 76 \\
\hline Yamagata & 73 & 74 & 71 & Wakayama & 64 & 66 & 68 \\
\hline Fukushima & 69 & 69 & 69 & Tottori & 72 & 72 & 75 \\
\hline Ibaraki & 73 & 72 & 74 & Shimane & 74 & 75 & 78 \\
\hline Tochigi & 71 & 67 & 68 & Okayama & 65 & 65 & 72 \\
\hline Gunma & 61 & 60 & 63 & Hiroshima & 64 & 67 & 65 \\
\hline Saitama & 66 & 63 & 63 & Yamaguchi & 70 & 74 & 78 \\
\hline Chiba & 68 & 64 & 67 & Tokushima & 66 & 67 & 71 \\
\hline Tokyo & 61 & 61 & 69 & Kagawa & 65 & 65 & 69 \\
\hline Kanagawa & 67 & - & 70 & Ehime & 62 & 67 & 70 \\
\hline Niigata & 72 & 73 & 74 & Kochi & 70 & 68 & 71 \\
\hline Toyama & 80 & 75 & 72 & Fukuoka & 65 & 66 & 73 \\
\hline Ishikawa & 69 & 70 & 70 & Saga & 67 & 71 & 73 \\
\hline Fukui & 77 & 74 & 72 & Nagasaki & 70 & 71 & 75 \\
\hline Yamanashi & 65 & 60 & 63 & Kumamoto & 70 & 70 & 75 \\
\hline Nagano & 73 & 72 & 73 & Oita & 66 & 69 & 72 \\
\hline Gifu & 64 & 64 & 65 & Miyazaki & 75 & 73 & 77 \\
\hline Shizuoka & 70 & 65 & 69 & Kagoshima & 71 & 70 & 75 \\
\hline Aichi & 64 & 64 & 65 & Okinawa & 74 & 73 & 74 \\
\hline Mie & 67 & 66 & 66 & mean (SD) & & $69(4)$ & \\
\hline
\end{tabular}


Table 4 Annual average concentration of photochemical oxidants by prefecture

\begin{tabular}{|c|c|c|c|c|c|c|c|}
\hline \multirow[t]{2}{*}{ Prefecture } & \multicolumn{3}{|c|}{ Photochemical oxidants (ppm) } & \multirow[t]{2}{*}{ Prefecture } & \multicolumn{3}{|c|}{ Photochemical oxidants (ppm) } \\
\hline & 2010 & 2013 & 2016 & & 2010 & 2013 & 2016 \\
\hline Hokkaido & 0.032 & 0.029 & 0.031 & Shiga & 0.038 & 0.037 & 0.036 \\
\hline Aomori & 0.032 & 0.032 & 0.033 & Kyoto & 0.036 & 0.035 & 0.034 \\
\hline Iwate & 0.029 & 0.030 & 0.031 & Osaka & 0.033 & 0.033 & 0.033 \\
\hline Miyagi & 0.032 & 0.030 & 0.033 & Hyogo & 0.032 & 0.033 & 0.033 \\
\hline Akita & 0.036 & 0.036 & 0.035 & Nara & 0.032 & 0.032 & 0.033 \\
\hline Yamagata & 0.032 & 0.032 & 0.035 & Wakayama & 0.033 & 0.036 & 0.035 \\
\hline Fukushima & 0.034 & 0.032 & 0.033 & Tottori & 0.033 & 0.035 & 0.037 \\
\hline Ibaraki & 0.035 & 0.032 & 0.032 & Shimane & 0.038 & 0.040 & 0.038 \\
\hline Tochigi & 0.035 & 0.032 & 0.032 & Okayama & 0.033 & 0.032 & 0.032 \\
\hline Gunma & 0.036 & 0.036 & 0.038 & Hiroshima & 0.032 & 0.033 & 0.034 \\
\hline Saitama & 0.034 & 0.033 & 0.032 & Yamaguchi & 0.034 & 0.035 & 0.035 \\
\hline Chiba & 0.031 & 0.032 & 0.032 & Tokushima & 0.032 & 0.033 & 0.038 \\
\hline Tokyo & 0.032 & 0.032 & 0.031 & Kagawa & 0.030 & 0.033 & 0.036 \\
\hline Kanagawa & 0.029 & 0.032 & 0.031 & Ehime & 0.032 & 0.033 & 0.033 \\
\hline Niigata & 0.037 & 0.036 & 0.037 & Kochi & 0.033 & 0.033 & 0.036 \\
\hline Toyama & 0.037 & 0.036 & 0.038 & Fukuoka & 0.031 & 0.032 & 0.035 \\
\hline Ishikawa & 0.037 & 0.036 & 0.039 & Saga & 0.035 & 0.036 & 0.036 \\
\hline Fukui & 0.035 & 0.037 & 0.037 & Nagasaki & 0.036 & 0.035 & 0.036 \\
\hline Yamanashi & 0.033 & 0.032 & 0.033 & Kumamoto & 0.034 & 0.033 & 0.035 \\
\hline Nagano & 0.035 & 0.034 & 0.035 & Oita & 0.032 & 0.031 & 0.035 \\
\hline Gifu & 0.031 & 0.033 & 0.035 & Miyazaki & 0.029 & 0.030 & 0.028 \\
\hline Shizuoka & 0.034 & 0.034 & 0.034 & Kagoshima & 0.030 & 0.031 & 0.032 \\
\hline Aichi & 0.031 & 0.032 & 0.033 & Okinawa & 0.029 & 0.028 & 0.031 \\
\hline Mie & 0.034 & 0.035 & 0.035 & mean (SD) & & $34(0.0$ & \\
\hline
\end{tabular}

\subsection{Multiple linear regression analysis}

Correlation analysis showed that ambient temperature, relative humidity, and photochemical oxidants were negatively correlated with smoking rate. As shown in Table 5, ambient temperature and relative humidity and photochemical oxidants are significant independent variables by stepwise multiple regression analysis using smoking rate as objective variables, ambient temperature, relative humidity and photochemical oxidants as explanatory variables. 
Table 5 Stepwise multiple regression analysis using smoking rate as objective variables, ambient temperature, relative humidity and photochemical oxidants as explanatory variables

\begin{tabular}{|c|c|c|c|c|c|c|}
\hline \multirow{2}{*}{$\begin{array}{l}\text { Independent } \\
\text { variables }\end{array}$} & \multirow{2}{*}{$\begin{array}{l}\text { Estimated } \\
\text { regression } \\
\text { coefficient B }\end{array}$} & \multicolumn{2}{|c|}{$95 \%$ confidence interval } & \multirow{2}{*}{$\begin{array}{l}\text { Partial regression } \\
\text { coefficient } \beta\end{array}$} & \multirow{2}{*}{$\begin{array}{l}\text { Cumulative } \\
\mathbf{R}^{2}\end{array}$} & \multirow{2}{*}{$\begin{array}{l}P- \\
\text { value }\end{array}$} \\
\hline & & Lower bound & $\begin{array}{l}\text { Upper } \\
\text { bound }\end{array}$ & & & \\
\hline Intercept & 40.817 & 34.965 & 46.668 & - & - & 0.000 \\
\hline $\begin{array}{l}\text { Ambient } \\
\text { Temperature }\end{array}$ & -0.464 & -0.580 & -0.349 & -0.555 & 0.247 & 0.000 \\
\hline $\begin{array}{l}\text { Photochemical } \\
\text { oxidants }\end{array}$ & -245.696 & -356.713 & -134.680 & -0.304 & 0.352 & 0.000 \\
\hline $\begin{array}{l}\text { Relative } \\
\text { Humidity }\end{array}$ & -0.067 & -0.129 & -0.006 & -0.154 & 0.375 & 0.031 \\
\hline
\end{tabular}

\section{Discussion}

Colder weather and fewer sunlight hours increase alcohol consumption [10]. This suggests that smoking may also be induced in cold and depressing environments. In addition, the author found that country latitudes significantly correlated with country smoking rates (unpublished, Figure 1) [11,12]. Climatic conditions such as cold and dry air are environmental stressors that can adversely affect human skin function [13-17]. In contrast, smoking temporarily relieves the stress of the cold. Nicotine administered through tobacco smoke and other delivery systems (eg, patch, nasal spray) produced acute analgesic effects that may be characterized as small to medium in magnitude [18-21]. Low humidity stimulates epidermal DNA synthesis and amplifies the hyperproliferative response to barrier disruption [22]. Smoking stimulates dopaminergic activity in the brain by inducing its release and inhibiting its degradation [23]. Dopamine D2-like receptor agonists accelerate barrier repair and inhibit the epidermal hyperplasia induced by barrier disruption [24]. On the other hand, photochemical oxidants and tobacco smoke are strong oxidative stressors [5, 25]. Combined oxidative stress can have serious adverse effects on smokers [26].

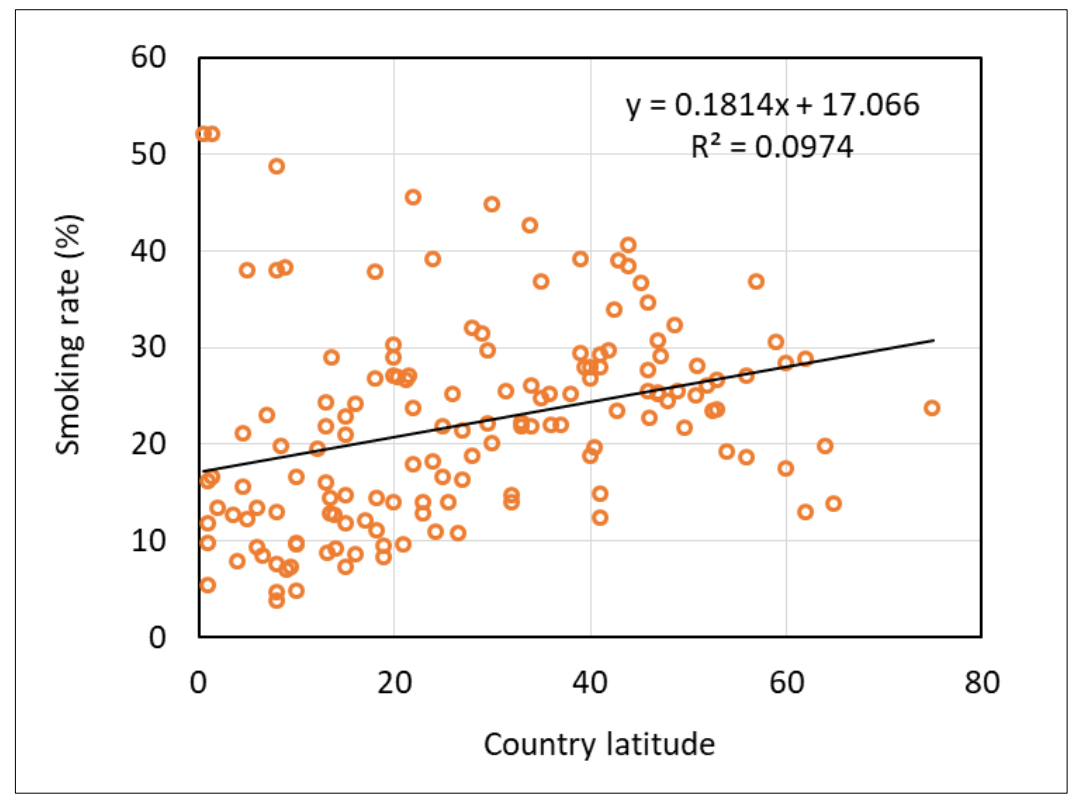

Figure 1 Correlation between latitude and smoking rate of countries in the world in 2018 


\section{Conclusion}

This result suggests that ambient temperature, relative humidity, and photochemical oxidants in the area may be related to smoking rate in the area. However, there is a limitation that the results of regression analysis cannot prove a causal relationship.

\section{Compliance with ethical standards}

\section{Acknowledgments}

The author appreciates the help of colleagues in the laboratory.

\section{Disclosure of conflict of interest}

There is no conflict of interest in this work.

\section{References}

[1] AD Revell, DM Warburton, K Wesnes. Smoking as a coping strategy. Addict Behav. 1985; 10(3): 209-224.

[2] M Hasenfratz, K Bättig. Psychophysiological interactions between smoking and stress coping? Psychopharmacology (Berl). 1993; 113(1): 37-44.

[3] Harwood GA, Salsberry P, Ferketich AK, Wewers ME. Cigarette smoking, socioeconomic status, and psychosocial factors: examining a conceptual framework. Public Health Nurs. 2007; 24: 361-371.

[4] Tina Jahnel, Stuart G. Ferguson, Saul Shiffman \& Benjamin Schüz, Daily stress as link between disadvantage and smoking: an ecological momentary assessment study. BMC Public Health. 2019; 19: 1284.

[5] Thomas Münzel, Andreas Daiber, Environmental sressors and their impact on health and disease with focus on oxidative stress. Antioxid Redox Signal. 2018, 28(9): 735-740.

[6] Lee Taylor, Samuel L. Watkins Hannah Marshall Ben Dascombe and Josh Foster, The impact of different environmental conditions on cognitive function: a focused review. Front Physiol. 2015; 6: 372.

[7] Toree L Bova, Ludovica Chiavaccini, Garrett F Cline, Caitlin G Hart, Kelli Matheny, Ashleigh M Muth, Benjamin E Voelz, Darrel Kesler, Erdoğan Memili. Environmental stressors influencing hormones and systems physiology in cattle. Reproductive Biology and Endocrinology. 2014; 12: 58.

[8] Japan Meteorological Agency, General information on climate of Japan. [Internet]. [cited 2021 May 24].

[9] S Suna. Pollutants and climatic conditions related to the formation of photochemical oxidants. World Journal of Biology Pharmacy and Health Sciences. 2021; 5(2): 1-5.

[10] Meritxell Ventura-Cots, Ariel E. Watts, Monica Cruz-Lemini, Neil D. Shah, Nambi Ndugga, Peter McCann, A. Sidney Barritt IV, Anant Jain, Samhita Ravi, Carlos Fernandez-Carrillo, Juan G. Abraldes, Jose Altamirano, and Ramon Bataller, Colder weather and fewer sunlight hours increase alcohol consumption and alcoholic cirrhosis Worldwide. Hepatology. 2019; 69(5): 1916-1930.

[11] WHO. Age-standardized estimates of current tobacco use, tobacco smoking and cigarette smoking- Data by country. [Internet]. [cited 2021 Sep 14].

[12] GPS Coordinates. Latitude and Longitude by Countries. [Internet]. [cited 2021 Sep 14].

[13] E Berardesca, M Farage, H Maibach. Sensitive skin: an overview, International Journal of Cosmetic Science. 2013; 35: 2-8.

[14] KA Engebretsen, JD Johansen, S Kezic, A Linneberg, JP Thyssen. The effect of environmental humidity and temperature on skin barrier function and dermatitis. J Eur Acad Dermatol Venereol. 2016; 30(2): 223-249.

[15] Tasuku Akiyama, Mirela Iodi Carstens, E Carstens. Spontaneous itch in the absence of hyperalgesia in a mouse hindpaw dry skin model. Neurosci Lett. 2010; 484(1): 62-65.

[16] Tlougan BE, Mancini AJ, Mandell JA, Cohen DE, Sanchez MR. Skin conditions in figure skaters, ice-hockey players and speed skaters: part II - cold-induced, infectious and inflammatory dermatoses. Sports Med. 2011; 41(11): 967-984. 
[17] Joseph W Ditre, Bryan W Heckman, Emily L Zale, Jesse D Kosiba, Stephen A. Acute analgesic effects of nicotine and tobacco in humans: a meta-nalysis. Pain. 2016; 157(7): 1373-1381.

[18] Benowitz NL, Nicotine and postoperative management of pain. Anesth Analg. 2008; 107(3): 739-741.

[19] Campbell VC, Taylor RE, Tizabi Y. Effects of selective opioid receptor antagonists on alcohol-induced and nicotineinduced antinociception. Alcohol Clin Exp Res. 2007, 31(8), 1435-1440.

[20] Ditre JW, Heckman BW, Butts EA, Brandon TH. Effects of expectancies and coping on pain-induced motivation to smoke. J Abnorm Psychol. 2010; 119(3): 524-533.

[21] R Merzak. Myofascial trigger points: relation to acupuncture and mechanisms of pain. Arch Phys Med Rehabil.1981 Mar; 62(3): 114-117.

[22] M Denda, J Sato, T Tsuchiya, PM Elias, KR Feingold. Low humidity stimulates epidermal DNA synthesis and amplifies the hyperproliferative response to barrier disruption: implication for seasonal exacerbations of inflammatory dermatoses. J Invest Dermatol. 1998; 111(5): 873-878.

[23] Marina Sagud, Alma Mihaljević-Peles, Dorotea Mück-Seler, Nela Pivac, Bjanka Vuksan-Cusa, Tomo Brataljenović, Miro Jakovljević, Smoking and schizophrenia. Psychiatr Danub. 2009; 21(3): 371-375.

[24] Shigeyoshi Fuziwara, Ayako Suzuki, Kaori Inoue, Mitsuhiro Denda. Dopamine D2-like receptor agonists accelerate barrier repair and inhibit the epidermal hyperplasia induced by barrier disruption. J Invest Dermatol. 2005; 125(4): 783-789.

[25] Reema Goel, Zachary Bitzer, Samantha Reilly, Neil Trushin, Lisa Reinhart, Ryan Elias, John P Richie, Tobacco smoke free radicals and related biomarkers of oxidative stress. Free Radical Biology and Medicine, Volume 112, Supplement. 1 November 2017; 130-131.

[26] Ashley Jerath Tatum, Gail G Shapiro. The effects of outdoor air pollution and tobacco smoke on asthma. Immunol Allergy Clin North Am. 2005; 25(1): 15-30. 